Check for updates

Cite this: RSC Chem. Biol., 2021, 2, 1631

Received 9th April 2021,

Accepted 18th August 2021

DOI: $10.1039 / \mathrm{d} 1 \mathrm{cb} 00081 \mathrm{k}$

rsc.li/rsc-chembio

\section{Origin, toxicity and characteristics of two amyloid oligomer polymorphs}

\author{
Chamani Niyangoda, ${ }^{a}$ Jeremy Barton, ${ }^{a}$ Nabila Bushra, ${ }^{a}$ Kanchana Karunarathne, ${ }^{a}$ \\ Graham Strauss, ${ }^{\mathrm{b}}$ Fadia Fakhre, ${ }^{\mathrm{a}}$ Piyush Koria ${ }^{\mathrm{b}}$ and Martin Muschol (D) *a
}

\begin{abstract}
There is compelling evidence that small oligomeric aggregates, emerging during the assembly of amyloid fibrils and plaques, are important molecular pathogens in many amyloid diseases. While significant progress has been made in revealing the mechanisms underlying fibril growth, understanding how amyloid oligomers fit into the fibril assembly process, and how they contribute to the pathogenesis of amyloid diseases, has remained elusive. Commonly, amyloid oligomers are considered to be metastable, early-stage precursors to fibril formation that are either on- or off-pathway from fibril growth. In addition, amyloid oligomers have been reported to colocalize with late-stage fibrils and plaques. Whether these early and late-stage oligomer species are identical or distinct, and whether both are relevant to pathogenesis remains unclear. Here we report on the formation of two distinct oligomer species of lysozyme, formed either during the early or late-stages of in vitro fibril growth. We further observe that the $\mathrm{pH}$ change from in vitro growth conditions to cell media used for toxicity studies induced distinct mesoscopic precipitates, two of which resemble either diffuse or neuritic plaques seen in Alzheimer's histology. Our biophysical characterization indicates that both oligomer species share morphological and tinctorial features considered characteristic for amyloid oligomers. At the same time, their sizes, morphologies, their immunostaining, detailed tinctorial profiles and, most prominently, their biological activity are clearly distinct from each other. Probing the conditions promoting the formation of these two distinct oligomer species suggests distinct roles of charge interactions, hydrophobicity and monomer flexibility in directing oligomer assembly.
\end{abstract}

\section{Introduction}

Amyloidoses refer to a group of human disorders all characterized by the deposition of protein fibril plaques appearing in different, disease-specific tissues and organs. ${ }^{1-4}$ While the proteins that form these amyloid plaques are unrelated and disease-specific, the resulting fibrils have a shared cross-beta sheet architecture. ${ }^{3,5}$ This structural commonality has been linked to the underlying propensity of polypeptide chains to form intermolecular hydrogen bonds across their backbone. ${ }^{5,6}$ However, the role of amyloid fibrils as the underlying cause of disease symptoms is much less clear. In several nonneuropathic amyloidoses fibril deposits have been implicated as the primary cause of disease pathology. ${ }^{7}$ Conversely, there is compelling evidence that small oligomers, emerging during the assembly of amyloid fibrils and plaques, are potent molecular pathogens particularly in neurodegenerative diseases. ${ }^{8-13}$

\footnotetext{
${ }^{a}$ Dept. of Physics, University of South Florida, Tampa, FL 33620, USA.

E-mail: mmuschol@usf.edu

${ }^{b}$ Dept. of Chemical and Biomedical Engineering, University of South Florida,

Tampa, FL 33620, USA
}

Establishing causality between amyloid formation and disease, however, has been complicated by recent findings that both fibrils and oligomers can exist in multiple polymorphic forms with distinct pathogenic potentials. ${ }^{14-18}$ In addition, there is evidence that different types of amyloid aggregates can interact with and alter the accumulation of one another. ${ }^{19-23}$ For Alzheimer's disease, there are strong indications that $\mathrm{A} \beta$ aggregates interact with tau, $\alpha$-syn and TDP-43, each of which is associated with distinct neurodegenerative diseases of its own, thereby adding to the complexity of amyloid species and their relation to pathogenesis. ${ }^{20,24}$

Detailed models of the molecular steps and mechanisms regulating fibril nucleation and growth, together with analytical solutions to the resulting kinetics of fibril assembly, have significantly enhanced our mechanistic understanding of amyloid fibril self-assembly. ${ }^{25,26}$ In contrast, the conditions and mechanisms inducing or modulating the formation of metastable amyloid oligomers, in vitro or in vivo, remain poorly understood. In addition, there is evidence for the presence of multiple, distinct oligomer polymorphs formed in vitro and in vivo. ${ }^{27-31}$ This raises the questions of how oligomer species come about, what distinguishes them from one another, which 
conditions promote the formation of one over the other, and how they fit into the overall fibril assembly process. Commonly, amyloid oligomers are considered to be metastable, early-stage precursors that are either on- or off-pathway from fibril growth. ${ }^{19,27,32,33}$ At the same time, late-stage fibrils and plaques have been implicated as reservoirs for amyloid oligomer formation. ${ }^{34}$ Whether those two modes of oligomer formation are identical or distinct, and whether both are relevant to pathogenesis is unclear. Understanding the mechanisms underlying the formation of various oligomer polymorphs, and how they relate to each other, would provide important insights into their contributions to pathogenesis in different cell types and tissues, and open up new strategies for therapeutic interventions. Similarly, elucidating the relation between oligomer to fibril formation directly affects the approaches taken for targeting oligomers $v s$. fibrils as pathogenic species in amyloid disease. ${ }^{35}$

Here we describe the formation of two distinct amyloid oligomer species formed in vitro by the amyloid protein lysozyme. Intriguingly, these two distinct amyloid species form under nearly identical growth conditions, but at different time points of the fibril assembly process. While one oligomer species assembles during the initial lag phase of fibril formation, the second population emerges at the late-stages of fibril growth. For the sole purpose of distinguishing these two oligomer species, we will refer to them as early-stage versus latestage oligomers (ESOs vs. LSOs). We had previously characterized ESOs as off-pathway competitors and inhibitors of fibril formation. ${ }^{19,23}$ We therefore focused on characterizing the sizes, morphologies and composition of LSOs, and determined their tinctorial and immunological signatures relative to those of fibrils and ESOs. Using a live/dead fluorescence assay we established that ESOs, LSOs and fibrils all display distinct levels of cell toxicity. We further observed that transfer from growth solutions to cell media induced prominent changes in morphology for each aggregate species, with fibrils and ESOs showing striking similarities to neuritic fibrillar $v s$. diffuse plaque associated with Alzheimer's disease. Tracking the relationship of fibrils to LSOs indicated that the latter, just like their ESO counterparts, emerge as off-pathway byproduct of fibril growth conditions. Our findings provide some mechanistic insights into how distinct oligomer species can emerge as result of compromises between intermolecular charge repulsion, hydrophobicity and structural flexibility.

\section{Results}

We have previously reported that in vitro amyloid assembly of lysozyme under acidic conditions proceeds along two distinct assembly pathways. One pathway is characterized by lag-free oligomer formation, but only emerges upon crossing a (protein and salt-dependent) threshold concentration. Due to its similarity with micelle formation by lipids, we refer to this threshold as 'critical oligomer concentration' or COC. The rate at which these metastable globular early-stage oligomers (ESOs)
A

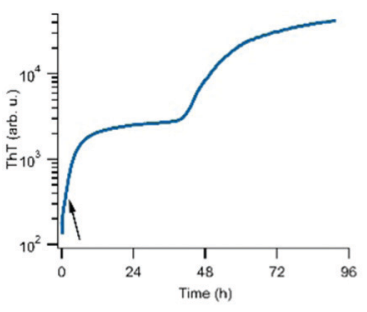

C

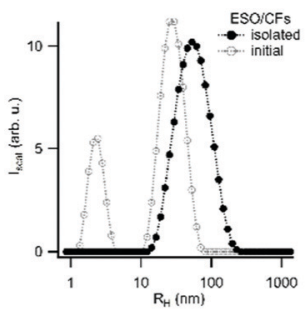

B

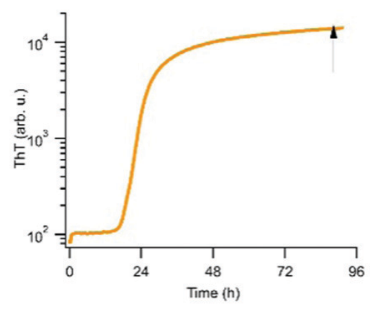

D

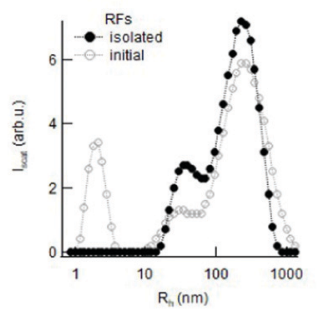

Fig. 1 Biphasic vs. sigmoidal ThT kinetics and distinct particle size distributions indicate early-stage oligomer (ESO) vs. rigid fibril (RF) dominated amyloid growth. (A) Biphasic ThT kinetics associated with formation of ESO/CFs during initial phase of amyloid assembly (1.4 mM hewL at $250 \mathrm{mM}$ $\mathrm{NaCl}$ ). (B) Typical sigmoidal ThT kinetics indicating RF dominated amyloid formation (1.4 $\mathrm{mM}$ hewL at $50 \mathrm{mM} \mathrm{NaCl}$ ). The small, initial step of ThT in B results from surface binding of denatured hewL upon heating. Both solutions were incubated at $\mathrm{pH} 2,52{ }^{\circ} \mathrm{C}$. The arrows indicate typical time points for the collection of either ESO/CFs or RFs for toxicity measurements (C) DLS particle size distribution (PSD) of early-stage oligomers (ESO) and curvilinear fibrils (CFs), after incubation (open circles), and after their isolation from monomers (filled circles). (D) Typical tri-modal PSD of hewL RFs grown under sigmoidal conditions, after incubation (open circles), and after their isolation from monomers (filled circles).

and curvilinear fibrils (CFs) form increases as a power law of the protein concentration above the threshold. The subsequent fibril nucleation event induces the biphasic ThT kinetics, which is characteristic for this pathway (Fig. 1A). ${ }^{19,36,37}$ Below the aforementioned threshold, the nucleated growth of amyloid filaments and fibrils (we collectively refer to as rigid fibril, or RFs) displays the ubiquitous sigmoidal ThT kinetics. During the extended lag phase of this pathway, no discernible populations of oligomeric precursors are observed. However, the cell toxicity experiments detailed below revealed that the fibril samples generated during sigmoidal fibril growth contained a second class of "late-stage" oligomers (LSOs). Here we isolated each of these oligomeric and fibrillar species for quantitative cell toxicity assays, and for determining their respective morphological, tinctorial, and immunological characteristics. We have previously characterized multiple features of the fibrils and ESO/CFs generated during sigmoidal and biphasic growth, respectively. We therefore focused on elucidating the origin of the LSOs formed during sigmoidal fibril growth, and on characterizing the new LSOs relative to RFs and ESOs.

\subsection{Growth and isolation of oligomeric and fibrillar amyloids}

Aggregates were generated under similar in vitro growth conditions, and without addition of any denaturants. In both cases, 
$1.4 \mathrm{mM}$ of hewL was dissolved in water adjusted to $\mathrm{pH} 2$ with $\mathrm{HCl}$, and was incubated at $T=52{ }^{\circ} \mathrm{C}$. Fibril- $v s$. oligomerdominated growth, as indicated by their respective ThT kinetics or particle-size distribution profiles (Fig. 1), was induced by using either $50 \mathrm{mM}$ or $250 \mathrm{mM} \mathrm{NaCl}$ in the solutions. ${ }^{19,36-38}$ The progress of the aggregation reaction was monitored using dynamic light scattering. The reaction was stopped at time points appropriate to obtain suspensions of rigid fibrils (RFs) and late-stage oligomers (LSOs), typically after 6-8 days at $50 \mathrm{mM}$ NaCl. Early-stage oligomers (ESOs), in turn, were harvested within 2-8 hours of incubation at $250 \mathrm{mM} \mathrm{NaCl}$. We have previously shown that ESOs have a strong tendency to self-assemble into large curvilinear fibrils (CFs) which become the dominant aggregate species present in solution. ${ }^{38,39}$ Transmission electron microscope images of the ESO/CFs grown under bimodal conditions show the dominant $\mathrm{CF}$ population (Fig. 2A). TEM images of RFs formed during sigmoidal growth, in turn, display the characteristic rigid fibril shapes (Fig. 2B). The cell toxicity studies described below indicated the presence of an additional "late-stage" oligomer (LSO) species formed during the prolonged sigmoidal RF growth. Fig. 2C shows AFM images of LSO following their isolation from the simultaneously present RFs.

For quantification of their cell toxicity, we isolated oligomeric and fibrillar aggregates from their monomeric background, and each other, using a combination of centrifugation and molecular weight cut-off filters. Fibrils were centrifuged overnight at $14000 \mathrm{~g}$ and the pellet re-suspended. This process was repeated three times in order to obtain a pure RF suspension. Successful removal of residual monomers and oligomers was confirmed by the absence of UV-absorption from the supernatant after the third spin, and by the loss of the monomer peak in DLS (Fig. 1D). The same approach was used to isolate ESO/CFs. Isolation of LSOs started with the fibril
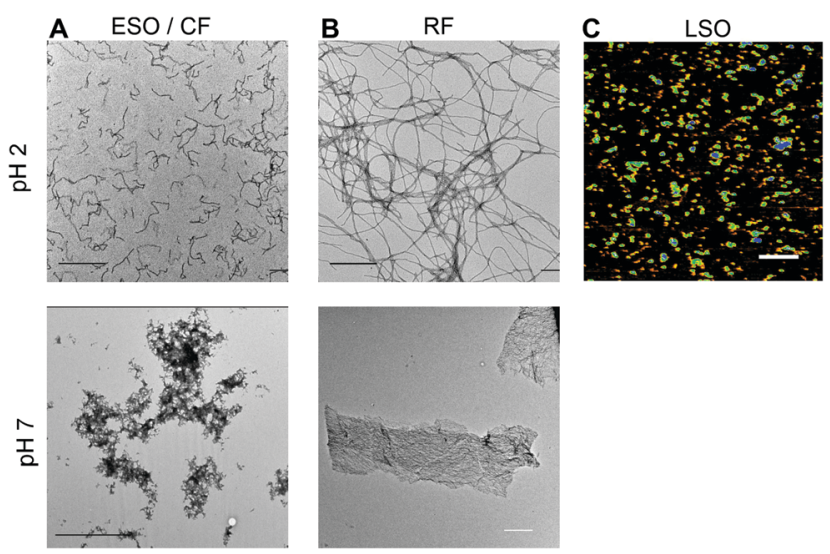

Fig. 2 Solution transfer of aggregates creates precipitates with distinct morphologies. (A) TEM images of separated early-stage oligomers (ESOs) and their polymerized curvilinear fibrils (CFs) formed at $\mathrm{pH} 2$ (top), and following transfer to $\mathrm{pH} 7$ (bottom). (B) TEM images of rigid fibrils (RFs) formed under sigmoidal growth conditions at $\mathrm{pH} 2$ (top) and after transfer to $\mathrm{pH} 7$ (bottom). (C) AFM image of late-stage oligomers, isolated from RFs after incubation at $\mathrm{pH}$ 2. Precipitates formed at $\mathrm{pH} 7$ were too large for imaging with AFM or TEM (scale bars: $500 \mathrm{~nm}$ ). supernatant obtained after the first centrifugation step. Residual fibrils were removed by passing the supernatant through a $50 \mathrm{~nm}$ syringe filter. Monomers were removed by passing this filtered supernatant through a $50 \mathrm{kDa}$ molecular weight cut off filter. This latter filtration step was repeated twice, with oligomers collected on top of the filter being re-suspended in solution. In all cases, we saw no significant aggregate dissociation into monomers over the course of our experiments. This implies that either the rates of dissociation are slow or monomer equilibria are very small. The effects of solution transfer discussed next only further shifted this balance towards the aggregated states.

\subsection{Formation of ordered $v s$. diffuse amyloid plaques upon solution transfer}

At $\mathrm{pH}$ 2, aggregates typically remained in suspension and displayed the morphologies of individual rigid or curvilinear fibrils (Fig. 2, top row). After transfer to $\mathrm{pH} 7.4$ cell medium, solutions consistently turned turbid indicating that all aggregate populations started to precipitate. The rate of precipitate formation and the resulting precipitate structures, however, were markedly different. RFs and ESO/CFs precipitated rapidly, while LSO precipitation evolved over several hours. RFs bundled into highly organized fibril sheets (Fig. 2B, bottom row). These bundles displayed bright ThS fluorescence and prominent birefringence in optical microscopy, without the need for Congo Red staining (Fig. 3, top row). Both features are considered diagnostic of amyloid plaques in vivo. ESOs and CFs, in contrast, precipitated into open-structured diffuse

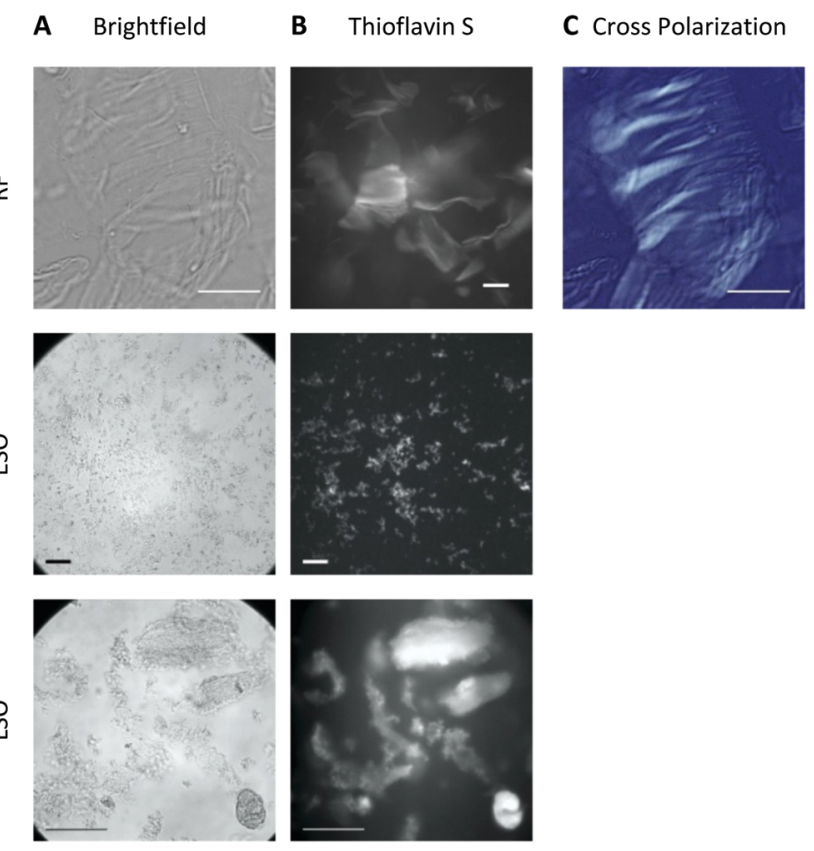

Fig. 3 Optical microscopy of amyloid precipitates formed at $\mathrm{pH} 7$. (A) Optical brightfield images (left column) of RFs (top), early-stage (middle) and late-stage oligomers (bottom). (B) Thioflavin $S$ fluorescence of aggregates in A (center column; intensities not calibrated). (C) Crosspolarized image of RF bundles. Scale bars: $100 \mu \mathrm{m}$. 
clusters (Fig. 2A, bottom row). These clusters exhibited no birefringence, indicating the lack of long-range alignment, retained their fractal-like morphology and showed only weak ThS affinity (Fig. 3, center row). Precipitates of LSOs formed compact aggregates too heavy for TEM imaging and, similarly to ESOs, displayed weak ThS fluorescence (Fig. 3, bottom row). The absence of optical activity again indicated the absence of long-range organization. These different amyloid precipitates showed noticeable differences in their affinity for cell membranes during the subsequent cell toxicity experiments. While fibril plaques strongly adhered to cell surfaces, both types of oligomeric precipitates could be mostly washed out following incubation.

The morphologies of the various hewL amyloid precipitates formed at $\mathrm{pH} 7.4$ resemble basic features of neuritic and dense core vs. diffuse plaques commonly observed in Alzheimer's disease and Down syndrome pathology. ${ }^{40-44}$ Both of these Alzheimer-associated plaques are composed of amyloid- $\beta$, with diffuse plaques often considered innocuous byproducts of $A \beta$ accumulation. Dense and neuritic plaques show intense ThS staining and optical birefringence while diffuse plaques only stain weakly, if at all, with ThS, and lack the long-range alignment required for optical birefringence. ${ }^{17,42,43,45,46}$ To our knowledge, though, the specific origin of diffuse plaques and their relation to fibrillar plaques has remained unresolved. The above observation with fibrillar vs. oligomeric precipitates suggests that the corresponding Alzheimer plaques might originate from these two distinct amyloid aggregate species and, therefore, provide insights into the progression of amyloid aggregation in vivo. In light of the toxicity data presented next, it is worth noting that diffuse plaques are not associated with tissue damage in $\mathrm{AD}$ or Down syndrome patients.

\subsection{Cell viability and altered cell morphology}

Cell viability measurements were performed with adenocarcinomic human alveolar basal epithelial (A549) cells using an imaging live-dead fluorescence assay. Upon growing cells to confluence, the cells were exposed to either isolated fibrillar or oligomeric aggregates. Our preliminary experiments revealed the presence of highly toxic, non-fibrillar species present in late-stage fibril solutions. We isolated the corresponding LSOs from their fibril background and included them as separate oligomer species in subsequent toxicity studies. Cells were exposed to amyloid aggregates ranging in concentrations from $1 \mu \mathrm{M}$ up to $100 \mu \mathrm{M}$, with the most prominent increase in toxicity between $5 \mu \mathrm{M}$ and $25 \mu \mathrm{M}$. Together with various controls, Fig. 4A shows the relative toxicity of the two oligomeric $v s$. fibrillar amyloid species at $5 \mu \mathrm{M}$ vs. $25 \mu \mathrm{M}$ concentration. We found the toxicity of hewL RFs to be quite modest. Since RF plaques did not readily wash out and were intensely stained by the ethidium homodimer and Hoechst 33342 dyes we could not evaluate the viability of A549 cells directly located underneath RF plaques.

Most striking, though, was the wide disparity in the relative toxicity of the two oligomeric species. While ESO/CF toxicity was comparable to the modest toxicity of RFs, LSOs were highly toxic. The imaging assay also revealed dramatic changes to the overall connectivity and organization of the confluent cell layer induced by LSOs. These were absent in cells exposed to either ESOs or RFs. Specifically, LSOs caused the formation of prominent voids in the confluent cell layer and a concurrent compaction of the remaining cells into misshapen clusters (Fig. 4B). The disruption of the overall connectivity by LSOs hints at their potential at disrupting epithelial cell layers in systemic amyloidoses and endothelial cell layers in vascular AD. It also
A

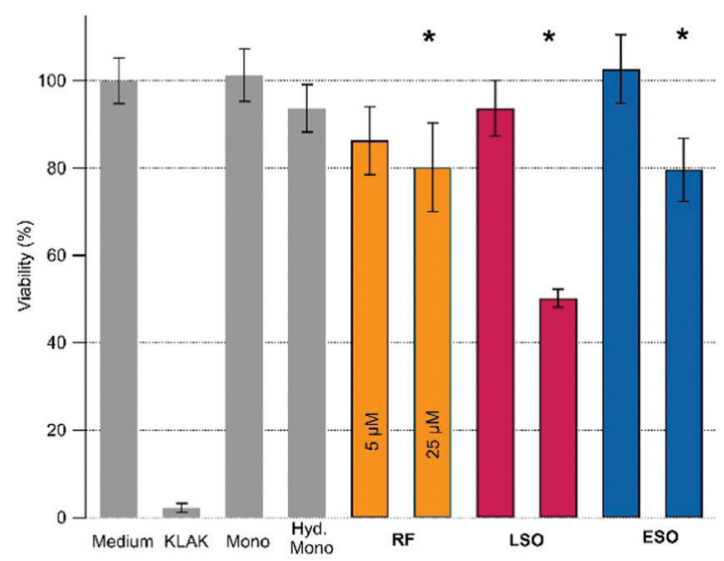

B

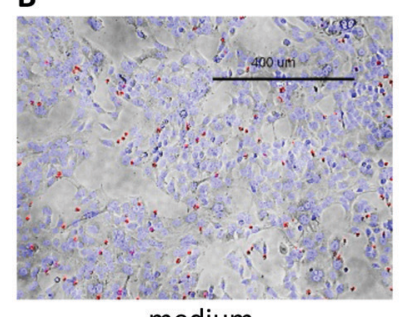
medium

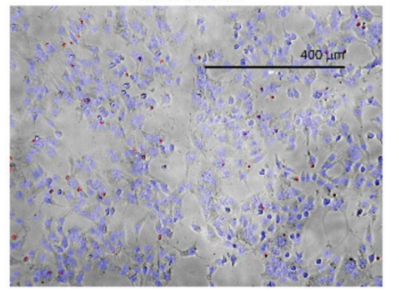

ESO

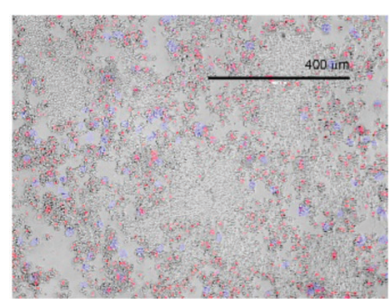

KLAK

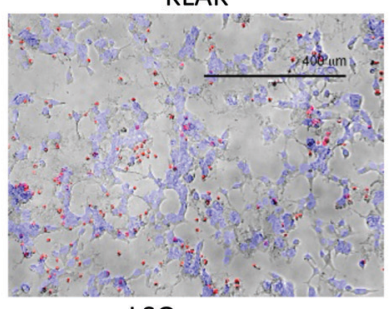

LSO

Fig. 4 Amyloid induced cell toxicity and altered cell morphology and connectivity. (A) Viability of A549 cells exposed to controls (cell medium, apoptotic toxin KLAK $(50 \mu \mathrm{M})$, native and hydrolyzed hewL monomers $(25 \mu \mathrm{M})$ as well as RFs, LSOs, and ESOs at 5 and $25 \mu \mathrm{M}$, each. Error bars indicate one standard deviation $(N=5)$. Stars indicate $p$-values $<0.05$. (B) Superposition of brightfield and fluorescence images of cells exposed to controls, ESOs or LSOs. Images for RFs were comparable to those of ESOs. LSOs, in contrast, altered both cell morphologies and disrupted cell layer connectivity, leading to islands of crowded cells with large voids in between. Scale bar: $400 \mu \mathrm{m}$. 
indicates that multiple amyloid oligomer species with distinct biological activity can form under conditions supportive of amyloid fibril formation.

\subsection{Origin of late-stage oligomers}

One obvious question is what causes the emergence of LSOs under growth conditions very similar to those used to generate ESOs, the latter of which we had characterized in our prior work..$^{36,37,39}$ In contrast to ESOs formed above the COC, neither ThT fluorescence nor dynamic light scattering measurements provided any indication for LSO formation during the lag phase of fibril growth under sigmoidal growth conditions (i.e. below the threshold for ESO formation). Toxic oligomers of IAPP and $\mathrm{A} \beta$ have been shown to be ThT negative but to form during the lag phase of fibril growth. ${ }^{32,47}$ We therefore explored whether hewL LSOs could have been formed throughout the assembly process but were invisible to ThT, or whether they were indeed late-stage products. In the latter case we wanted to determine whether LSOs emerged as direct result of fibril formation or were off-pathway from it. To probe the correlation between LSO and fibril formation, we utilized our observation that incubating solutions at decreasing surface-to-volume ratios accelerated fibril nucleation and growth, most likely due to preferential fibril nucleation at the air-water interface.

We monitored the time course of LSO formation during fibril growth conditions identical to those used for cell toxicity experiments. To probe the correlation between RF and ESO formation, we incubated two identical sets of seven $1.4 \mathrm{mM}$ hewL solutions at $50 \mathrm{mM} \mathrm{NaCl}$. One set of solutions was incubated in individual $1.5 \mathrm{ml}$ centrifuge tubes while the second set was distributed across 42 wells of a 96 well plate, filled with $250 \mu \mathrm{l}$ of solution each. Each day, the incubation of one centrifuge tube and six $250 \mu$ l wells was stopped, with the latter combined into one $1.5 \mathrm{ml}$ solution. LSO populations from these identical solution volumes were isolated following the same separation protocol used for cell toxicity measurements, and their concentrations measured using UV-absorbance. For the $1.5 \mathrm{ml}$ solutions, we had observed that fibril nucleation and growth required at least 4 days of incubation. Incubation of the $250 \mu \mathrm{l}$ wells in a fluorescence plate reader, in turn, allowed us to monitor the time course for fibril growth with $\mathrm{ThT}$ and relate it directly to the emergence of late-stage oligomers. Fig. 5A shows the time course for the build-up of LSOs in the single $1.5 \mathrm{ml} v s$. six $250 \mu \mathrm{l}$ incubation volumes. The ThT signal monitoring RF fibril formation in the 96 well plates is shown as well. In both incubation volumes the onset of toxic oligomer formation is delayed by several days, with LSO concentrations increasing rapidly thereafter. LSOs formed at $1.5 \mathrm{ml}$ solution volume do not become detectable until about 4 days of incubation, which is comparable to the onset of noticeable fibril formation under those conditions. In the 96 well plates, fibril growth rapidly accelerated already after 2 days. This, instead of accelerating, delayed and depressed LSO formation.

In addition, we confirmed that LSOs neither emerged as depolymerization products of fibrils, nor did fibrils catalyze LSO formation from monomers. To do so, we seeded isolated RFs
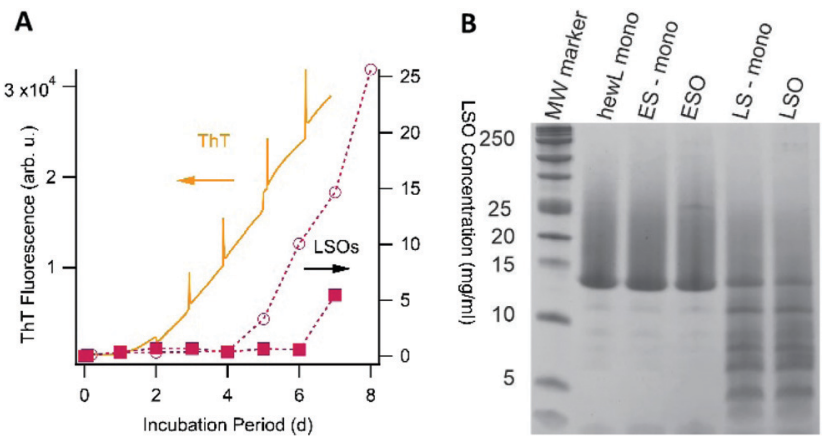

Fig. 5 LSOs are off-pathway and are composed of full-length, partially hydrolyzed monomers. (A) Two sets of seven lysozyme solutions were incubated under identical fibril/LSO growth conditions, but at different volumes $(1.5 \mathrm{ml}$ or $250 \mu \mathrm{l})$, resulting in different lag phases for fibril formation. Each day one $1.5 \mathrm{ml}$ solution and six $250 \mu \mathrm{l}$ wells were removed, the solution from the $250 \mu \mathrm{l}$ wells combined, and their oligomer content measured. LSO formation in the $1.5 \mathrm{ml}$ volume (open circles) commenced after 4 day, comparable with the lag period required for fibril formation under those conditions. Fibril formation in the plate reader (monitored via ThT, orange trace) started after just two days, with LSOs (filled squares) only emerging at day 7. (B) Reducing SDS-PAGE gel of freshly dissolved lysozyme monomers (hewL-mono), isolated early stage monomers (ES-mono) and oligomers (ESO), and isolated late-stage monomers (LS-mono) and oligomers (LSO). MW markers (in kDa) are shown as reference. The identical intensity of LS-mono and LSO bands indicates that LSOs are not composed of a specific fragment but contain the nicked but full-length LS-monos.

either in buffer alone (de-polymerization) or in buffer with added hewL monomers (catalytic oligomer formation) and incubated them under RF growth conditions for a time period of two days. This period was short enough to avoid "spontaneous" LSO formation from monomers. However, neither approach resulted in any detectable LSO formation (data not shown). Combining the above results, we conclude that toxic oligomers are neither fibril precursors, nor fibril depolymerization products. In addition, fibrils do not catalyze toxic oligomer formation from monomers. These experiments imply that LSO represent late-stage, off-pathway amyloid oligomers emerging under fibril growth conditions, and competing for their shared pool of monomers to grow from. Hence, just as we had established previously for ESOs, LSO formation is offpathway from fibril growth. However, they are distinct in their biological activity from off pathway ESOs formed under biphasic growth conditions.

The above data raise the question what causes the formation of this distinct oligomer species, and how to explain their delayed onset? One possibility is that LSOs form from either partially or fully hydrolyzed hewL monomers. ${ }^{37,48}$ HewL hydrolysis progresses slowly but steadily at the acidic $\mathrm{pH}$ and $52{ }^{\circ} \mathrm{C}$ incubation temperature used in our experiments. If so, LSOs might form because the monomers, while still tethered by their disulfide bonds, become partially nicked and acquire enhanced structural flexibility. ${ }^{48}$ Alternatively, LSOs might form from a fully hydrolyzed hewL fragment. To test these two alternatives, we determined the composition of LSOs and ESOs $v s$. the hydrolyzed monomers present at the same time point with 
SDS-PAGE under reducing conditions. Using reducing conditions identifies all hydrolyzed fragments, whether still tethered by disulfide bonds or not. Consistent with our prior observation, Fig. 5B indicates that ESOs form from the mostly intact monomers present during the early phases of incubation. ${ }^{37}$ In contrast, hewL monomers present during LSO formation are significantly hydrolyzed. Nevertheless, LSOs display the same distribution of hydrolysis fragments as the monomers present in solution. If a fully cleaved monomeric fragment were the building block for LSOs, this fragment would become enriched in LSOs. This implies that LSOs are formed from full-length but partially nicked monomers.

\subsection{LSO characterization}

Given the dramatic difference in the toxicity of ESOs vs. LSOs, we explored some of the basic biophysical, morphological, tinctorial, and immunological characteristics of this oligomer species in order to contrast it with the previously identified ESOs.

Size of late-stage oligomers. We performed DLS, FPLC, FTIR and immunological analyses on the late-stage monomeric and oligomeric components of the fibril supernatant (SN). DLS of the first $\mathrm{SN}$ after separation from fibrils indicated the presence of a small fraction of residual fibrils at about $100 \mathrm{~nm}$ diameter. Filtration through a $50 \mathrm{~nm}$ syringe removed these residual fibrils (Fig. 6A). The remaining "monomer peak" was shifted towards a hydrodynamic radius slightly larger than a pure monomer sample. This suggested the presence of an admixture of small oligomers and/or monomers enlarged by partial hydrolysis, which can't be resolved by DLS. Passing the same solution over a size exclusion chromatography column separated the supernatant into its monomeric and oligomeric components. All samples showed a monomer peak at $19.5 \mathrm{ml}$ and a weak shoulder or additional small peak towards lower molecular weights (Fig. 6B). This LMW shoulder/peak was likely due to the acid hydrolysis of a fraction of hewL monomers under these conditions. ${ }^{37,49}$ More importantly, the elution profile confirmed the presence of a significant oligomeric fraction in the supernatant - consistent with our toxicity studies using MW cut-off filters. Calibration using trypsin, pepsin and albumin indicated hewL dimers and trimers as the dominant oligomer species in the RF supernatant. These oligomers were noticeably smaller than the ESOs estimated to be octomers. ${ }^{39}$

Spectroscopic and immunological features. We have previously reported that the FTIR spectra suggested that hewL ESOs have an anti-parallel $\beta$-sheet structure. ${ }^{37}$ Fig. 6C shows the amide-I band for hewL LSOs and monomers obtained using attenuated total reflectance Fourier-transform infrared spectroscopy (ATR-FTIR). Just as ESOs, LSOs display peaks in the characteristic "amyloid $\beta$-sheet band" between 1610 and $1630 \mathrm{~cm}^{-1}$ wavenumbers, as well as indications of an additional peak near $1690 \mathrm{~cm}^{-1} \cdot{ }^{50}$ The latter is more apparent in the difference spectra (Fig. 6D) which are obtained by subtracting the area-matched spectra of monomers from those of LSOs. The wavelength band from $1680-1700 \mathrm{~cm}^{-1}$ is considered indicative of antiparallel $\beta$-sheet structures, and has been observed with oligomers from multiple amyloid proteins, including amyloid- $\beta$ and $\alpha$-synuclein. ${ }^{51-54}$ Antiparallel $\beta$ sheets have also been identified in the few high-resolution oligomer structures obtained with short peptide fragment of $\beta$-crystallin and human prion protein. ${ }^{55,56}$

Staining with the oligomer-selective antibody A11 antibody is a common assay to confirm the presence of amyloid oligomers. $^{57,58}$ For the dot-blots, the amount of hewL RFs deposited had to be titrated to account for the much stronger absorption and subsequent retention of RFs on the nitrocellulose membrane than for all other protein samples (see Methods for details). A11 did show noticeable cross-reactivity with hewL RFs, which we presume is due to the same non-specific stickiness the fibrils displayed for the nitrocellulose membrane, and for cells in the toxicity assay. Nevertheless, the A11 antibody preferentially recognized LSOs and reacted only very weakly
A

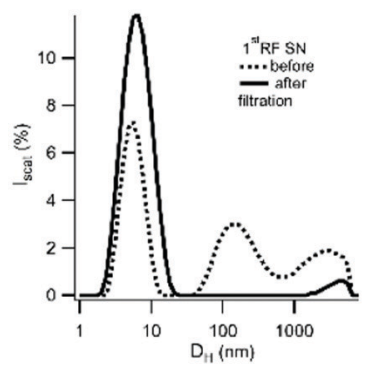

B

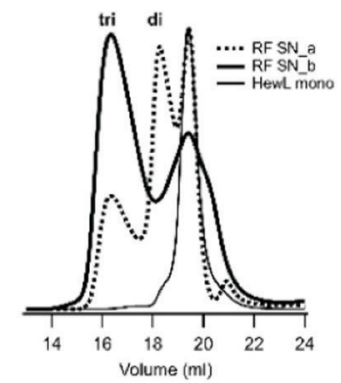

C

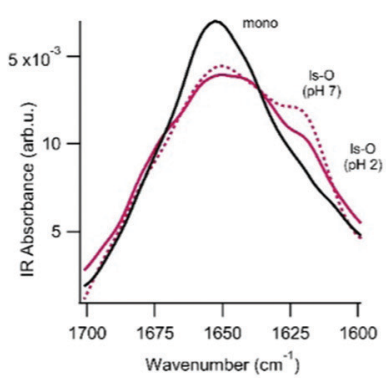

D

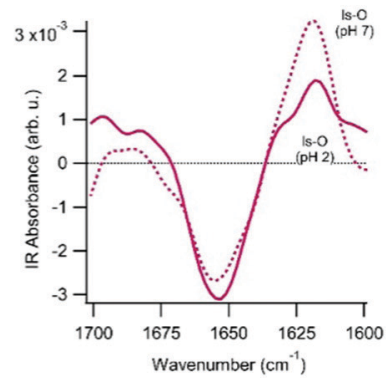

Fig. 6 Size and structural features of LSOs. Characterization of 1st supernatant (SN) from centrifugation of RFs grown under sigmoidal growth conditions. (A) DLS particle size distribution before (dotted line) and after (solid line) filtration through a 50 nm syringe filter to remove residual fibrils. The hydrodynamic diameter $D_{H}$ of the first peak suggests it contains a mixture of hewL monomers and small oligomers (B) FPLC of 1st supernatant (SN) from hewL fibrils grown for either (a) 6 or (b) 8 days. Dependent on the incubation period, one or two larger aggregates peaks emerge. Both SNs show a monomer peak and some hydrolyzed fragments. Aggregate peaks are consistent with hewL dimers (di) and trimers (tri). (C) Amide-I band of the FTIR spectra for LSO grown at $\mathrm{pH} 2$ and after transfer to $\mathrm{pH}$ 7, compared to hewL monomers. (D) Difference spectra of LSOs following subtraction of monomer reference spectra. A positive/negative value indicates an increase/decrease over the corresponding value over the monomer reference spectrum. 


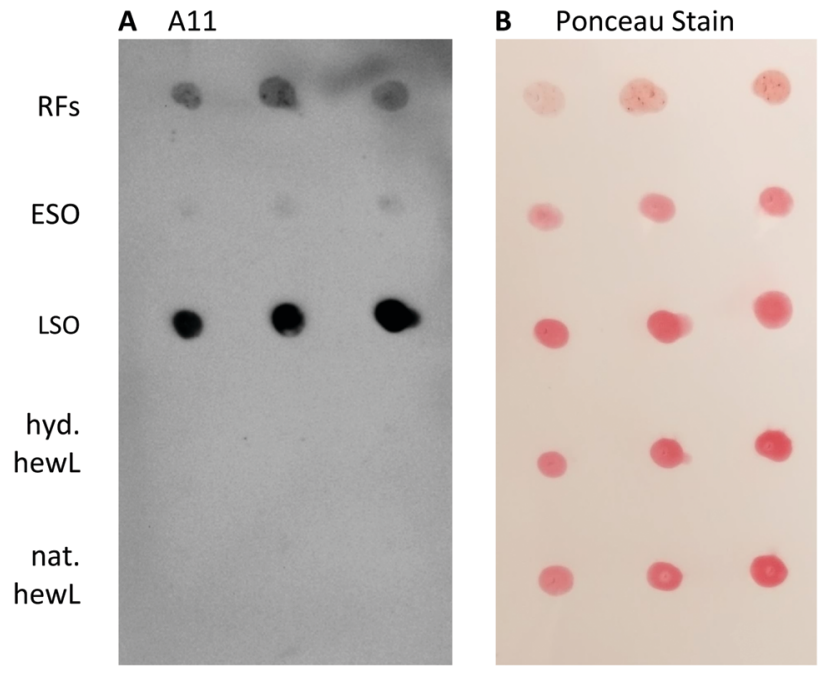

Fig. 7 Immuno-staining of early vs. late stage amyloid oligomers. Dot blots of different amyloid aggregates and controls at three different concentrations. (A) Reactivity of A11 with RFs (top row) and late-stage oligomers (middle row). (B) Ponceau staining of all samples blotted onto membrane. Concentrations for the various samples that yielded equivalent protein retention were (from left to right) RFs: $2.0,1.0$ and $0.5 \mu \mathrm{g}$; all others: 12,8 and $4 \mu \mathrm{g}$.

with ESOs (Fig. 7). This lines up with the difference in cellular toxicity and supports an underlying structural difference between these two oligomer species.

\section{Tinctorial profiles}

Recently, differences in the emission of multiple fibril-specific dyes have been used to identify different fibril polymorphs isolated from patients with familiar $v s$. sporadic Alzheimer's. ${ }^{59}$ Here we extended this approach by using a combination of fibril-selective (ThT, FSB and curcumin) and oligomer-sensitive (bis-ANS, crystal violet) fluorescent dyes to determine the fluorescence profiles of ESOs and LSOs relative to those of hewL RFs. Bis-ANS has been recognized as oligomer-selective by multiple investigators. ${ }^{60-62}$ Our lab recently identified crystal violet as another oligomer-selective dye. ${ }^{63}$ As expected, the fibril-selective amyloid dyes all showed significantly higher fluorescence enhancements upon binding to RFs than to ESOs or LSOs at the same concentration (Fig. 8). While quite different in amplitude to RF responses, fibril dye fluorescence was enhanced by both LSOs and ESOs. This suggests that fibrils and oligomers do share some structural characteristics, most likely the formation of intermolecular hydrogen bonds. The modest but noticeable ThT response evoked by LSOs, combined with the absence of a ThT increase in the lag phase of sigmoidal fibril growth, further supports our contention that LSOs are absent during the lag phase of sigmoidal fibril growth. In contrast to the fibril-selective dyes, fluorescence responses of bis-ANS and crystal violet to ESOs and LSOs were similar to those evoked by RFs. The significant hydrophobicity of RFs might underlie their inherent tendency to form sheet-like plaques upon their decrease in charge repulsion with
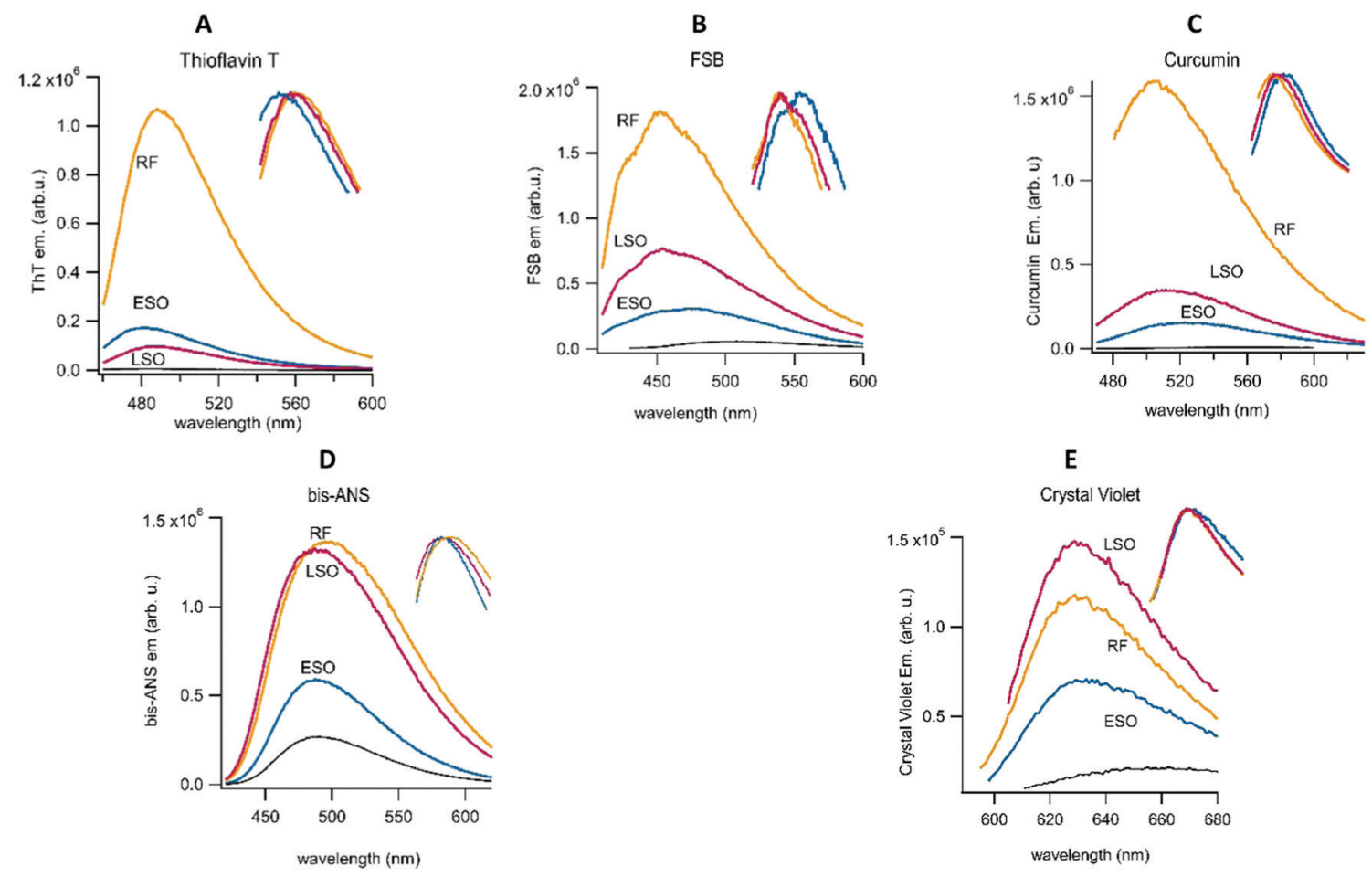

E

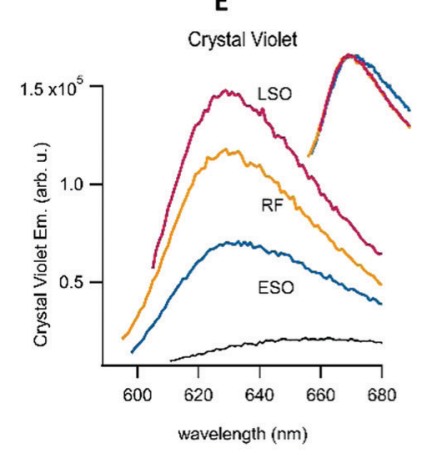

Fig. 8 Responses of fibril- vs. oligomer-sensitive dyes to RFs, ESOs and LSOs. Fluorescence emission spectra of (A) the amyloid indicator dyes thioflavin $\mathrm{T}$ (B) the fluorescent Congo Red derivative FSB and (C) the amyloid binding dye curcumin (D) the environment-sensitive dye bis-ANS and (E) the oligomer dye crystal violet. Dye and aggregate concentrations were typically $15 \mu \mathrm{M}$ and $1 \mathrm{mg} \mathrm{ml}^{-1}$, respectively. Monomer reference spectra are shown in black. Spectral shifts to the emission peaks are highlighted in the insert to each spectrum. 
increasing pH. For all dyes, though, ESOs and LSOs show clearly distinguishable fluorescence enhancements. Several of the dyes also display modest but noticeable changes in their peak emission wavelength or spectral shape in the presence of the three aggregate species (see inserts). The enhanced response to LSOs vs. ESOs by the environmentally sensitive dye bis-ANS and crystal violet points to an underlying difference in the hydrophobicity of these two oligomeric species that likely correlates with their noticeable differences in cell toxicity.

\section{Discussion and conclusion}

Our experiments indicate that slight changes in the conditions supportive of amyloid fibril formation by lysozyme can generate two distinct conformers of amyloid oligomers we have labelled early-stage (ESOs) and late-stage oligomers (LSOs). ESOs emerge as "precursor" to fibrils during the lag phase of fibril formation. We have previously shown that the process of ESO formation in lysozyme is identical to that observed with amyloid- $\beta$, that they are off-pathway from fibril formation, and that their emergence requires the crossing of a threshold concentration we called the "critical oligomer concentration" or COC. ${ }^{19,36}$ We further confirmed that ESOs and curvilinear fibrils (CFs) of both $\mathrm{A} \beta$ and lysozyme actively inhibit secondary nucleation of amyloid fibrils by binding to the lateral surfaces of fibril seeds. ${ }^{23}$ In contrast to ESOs, LSOs require extended incubation at concentrations below the COC of ESOs. For some other amyloid proteins, such as IAPP, toxic oligomers emerged without ThT response during the lag phase of fibril growth. LSOs formed by hewL, instead, did not form during the lag phase of fibril growth. ${ }^{47}$ While overlapping with the time course for fibril formation, our time-resolved measurements of RF vs. LSO formation indicate that LSOs, just as their ESO counterparts, are forming off-pathway during fibril growth. Reducing SDS-PAGE indicates that ESOs and LSOs are both composed of full-length lysozyme instead of some hydrolysisinduced peptide fragment. However, while ESOs assemble from essentially intact monomers, LSOs are formed from severely nicked monomers. This matches with prior results documenting hydrolysis effects on lysozyme fibril formation upon extended incubation at $\mathrm{pH} 2 .^{48}$

Isolating each of the three different amyloid aggregate species (ESOs, LSOs and RFs) from monomers, and from each other, allowed us to assess their relative cell toxicity. Interestingly, while ESOs and RFs were only mildly noxious, LSOs proved to be highly toxic to cells. There are two prior reports of lysozyme oligomers formed after weeks of incubation under acidic growth conditions. These oligomers were shown to be highly toxic to pheochromocytoma (PC12) cells ${ }^{64}$ and to induce toxicity and tau hyperphosphorylation in cortical neuronal slices at levels comparable to A $\beta 42$ oligomers. ${ }^{65}$ We suspect that these oligomers were similar to the LSOs generated here. However, the growth temperature and incubation periods were sufficiently different to prevent a direct comparison.
The authors also did not indicate whether $\mathrm{pH}$ transfer affected the structure of their aggregates.

The interpretation of aggregate toxicity is complicated by the inherent changes in aggregate structures upon transfer from pH 2 growth conditions to cell medium, but also provide some intriguing parallels to AD plaques. The precipitates of hewL RFs and ESOs closely resemble the morphological, optical and tinctorial characteristics of neuritic $v s$. diffuse plaques seen in post-mortem AD histology. The fact that hewL ESOs emerge as precursors to RFs also matches with the observation that diffuse plaques are considered benign precursors of late-stage neuritic plaques. ${ }^{43}$ At the same time, the rapid assembly of RFs and $\mathrm{ESO} / \mathrm{CFs}$ into larger structures might underlie the low levels of resulting cytotoxicity. Imaging the morphology of the cell layer revealed that LSOs not only were considerably more toxic than either RFs or ESOs, it also indicated that the former significantly altered cell morphology and cell layer connectivity. This effect suggests that amyloid toxicity measurements should not only account for single-cell responses but for the changes in tissue structures often seen in pathology. It is worth mentioning that the precipitation of LSOs proceeded relatively slowly, suggesting that significant populations of small oligomers remained in solution during cell exposure. While we suspect that the underlying structural differences between ESOs and LSOs are the main reason for their distinct cell toxicity, we can't exclude that the precipitation and differences in solubility upon transfer to cell medium contribute to the observed differences in cell toxicity. It is interesting to note, though, that amyloid formation in cells is likely to involve different cellular compartments with distinct environments. Hence those differences in morphology under different growth conditions might well be physiologically relevant.

Generating two oligomer polymorphs with distinct biological activity allowed us to investigate to what extend they share, or differ in, various characteristics associated with amyloid oligomers, and to explore the mechanism(s) promoting their formation. The molecular weights of both oligomer species fall in the range of just a few monomers, with ESOs estimated to contain eight monomers, ${ }^{39}$ while FLPC of LSOs suggested that they are as small as two to three monomers. Among the characteristics distinguishing these oligomers are their relative propensities towards further polymerization. While ESOs readily polymerize into curvilinear fibrils, ${ }^{39}$ also often referred to as protofibrils, LSOs did not form any discernible polymeric assemblies under their acidic growth conditions. Similar to our prior observations with ESOs, the infrared spectra of LSOs do contain amyloid-like intermolecular hydrogen bonds, as indicated by their peaks in the amyloid region $\left(1610-1630 \mathrm{~cm}^{-1}\right)$ of the amide-I bands. ${ }^{50}$ Equally important, a weak peak around $1680-90 \mathrm{~cm}^{-1}$, considered indicative of anti-parallel $\beta$-sheets, is present as well. ${ }^{51,52}$ In contrast to populations of ordered $v s$. disordered oligomers observed with $\alpha$-synuclein, and of disordered oligomers of $A \beta 40,{ }^{66,67}$ both LSOs and ESOs appear to be structured.

The fluorescence responses of fibril-selective $v s$. oligomersensitive dyes support several conclusions. Both ESOs and LSOs induce relatively muted fluorescence enhancements of 
fibril-selective dyes. At the same time, the fluorescence of the oligomer-specific dyes bis-ANS and crystal violet show robust enhancements in the presence of ESOs and LSOs. This pattern is characteristic of amyloid oligomers, in general, which are structurally distinct from amyloid fibrils. Equally important, LSOs consistently evoke more prominent fluorescence responses from both crystal violet and bis-ANS than ESOs. The enhanced response to bis-ANS and the smaller size of LSOs $v s$. ESOs matches with suggestions that increased hydrophobicity and reduced size of amyloid oligomers correlates with increases in cell toxicity. ${ }^{68,69}$ The difference in LSO vs. ESO structure and size as underlying cause for their distinct cellular toxicity is further buttressed by the preferential response of the anti-oligomer antibody A11 to LSOs.

Our in vitro experiments indicate that two distinct amyloid oligomer species can emerge under nearly identical solution conditions. This, in turn, raises the question what mechanisms regulate the formation of these distinct oligomer species? In the case of ESOs, we had previously shown that (a) lysozyme needed to be partially unfolded, and (b) ESO formation was limited by a protein/salt-dependent threshold related to the inherent charge repulsion among the monomers. ${ }^{36}$ In contrast, LSOs require significant partial hydrolysis of lysozyme monomers. We hypothesize that it is this increased flexibility and concurrent exposure of hydrophobic residues of the partially hydrolyzed monomers that are essential requirements for the assembly into LSOs. Interestingly, the point mutants of lysozyme implicated in human lysozyme amyloidosis, while structurally identical to the native state, also display increased structural flexibility and reduced thermal stability. ${ }^{70}$ The partial hydrolysis of hewL monomers under our growth conditions seems to provide an alternative way to promote such structural flexibility. This would imply that the flexibility of the amyloid monomer plays an important role in the type of amyloid oligomers that will assembly from them. Our data suggested that LSO are formed exclusively from hydrolyzed monomers and not as depolymerization product of fibrils. However, we can't exclude that prolonged incubation of RFs or changes in solution conditions, as described for $\beta$-microglobulin, could result in the release of monomers capable of forming "plaque-associated" LSOs. ${ }^{71}$ It is intriguing that folded proteins implicated in amyloidoses are all extracellular, and therefore more prone to degradation and enhanced structural flexibility during their life cycle.

Overall, the ability to generate two distinctly different and homogeneous oligomer populations without the use of denaturants or drugs can provide insights into the in vivo conditions promoting the formation of either relatively innocuous or highly toxic oligomer species. This could open up new approaches in targeting cellular pathways underlying pathogenic oligomer formation.

\section{Materials and methods}

\subsection{Chemicals and cell lines}

Two times recrystallized, dialyzed and lyophilized hen egg white lysozyme (hewL) was purchased from Worthington
Biochemicals (Lakewood NJ). Ultrapure grade thioflavin $\mathrm{T}$ (ThT) was obtained from Anaspec (Freemont, CA). Bis-ANS was obtained from Tocris Bioscience (Minneapolis, MN) and FSB from Calbiochem (San Diego, CA). Other chemicals were from Fisher Scientific (Pittsburgh, PA) and were reagent grade or better. All solutions were prepared using $18 \mathrm{M} \Omega$ water from a reverse osmosis unit (Barnstead E-pure, Dubuque, IA). A549 cells for toxicity were obtained from American Type Culture Collection (Manassas, VA).

\subsection{Growth and separation of amyloid aggregates}

The amyloid aggregate species generated from hewL included rigid fibrils (RFs), early-stage oligomers (ESOs) and their associated curvilinear fibrils (CFs), as well as late-stage oligomers (LSOs) were all formed at acidic $\mathrm{pH}$. Solution $\mathrm{pH}$ was estab-

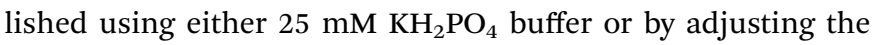
$\mathrm{pH}$ of the water/protein/salt solutions to $\mathrm{pH} 2$ by adding small amounts of hydrochloric acid. hewL was at a fixed concentration of $1.4 \mathrm{mM}$ in the $\mathrm{pH} 2,50 \mathrm{mM} \mathrm{NaCl}$ solution (for formation of RFs and LSOs) or $250 \mathrm{mM} \mathrm{NaCl}$ (for formation of ESO/CFs). Solutions were filtered through $220 \mathrm{~nm}$ PVDF syringe filters (Fisherbrand, Fisher Scientific) prior to incubation at $52{ }^{\circ} \mathrm{C}$. Protein samples with $50 \mathrm{mM} \mathrm{NaCl}$ were incubated for 6-8 days for $\mathrm{RF}$ formation while $250 \mathrm{mM} \mathrm{NaCl}$ hewL solutions were incubated for 2-8 hours and ESO/CF formation monitored with dynamic light-scattering (DLS). For quantification of their toxicity, individual aggregate species were isolated from monomers via centrifugation and/or use of molecular weight cut-off (MWCO) filters.

Isolation of fibrils. $\mathrm{RF}(50 \mathrm{mM} \mathrm{NaCl})$ solutions $(2 \mathrm{ml})$ were spun for 20-24 hours at $15000 \mathrm{~g}$ and $15{ }^{\circ} \mathrm{C}$. The RF supernatant (SN) and RF pellets were collected. Aggregate pellets were re-suspended in their respective solutions and spun again for 22-24 hours. This process was repeated until the monomer concentration in the supernatant was less than $1 \%$ of the aggregate concentration in the pellet. This typically required 3 cycles.

Isolation of LSOs. The LSOs present in the first RF supernatant were isolated from hewL monomers using $50 \mathrm{kDa}$ molecular weight centrifugal cut-off filters (Millipore Sigma Amicon, Fisher Scientific). The $50 \mathrm{kDa}$ pore size was required for passage of hewL monomers, which were retained by $30 \mathrm{kDa}$ filters. ${ }^{72} 500 \mu \mathrm{l}$ of the first RF SN was placed in the MWCO filter, spun at $15000 \mathrm{~g}$ for $20 \mathrm{~min}$. The filtrate after the first spin was collected for subsequent isolation of hydrolyzed monomers. The residue on top of the filter was washed adding $300 \mu \mathrm{l}$ of $50 \mathrm{mM} \mathrm{NaCl} \mathrm{pH} 2$ solution. After three washes, the filter was flipped, put into a new centrifuge tube and spun at $1000 \mathrm{~g}$ for 5 minutes to collect isolated toxic oligomers.

Isolation of hydrolyzed monomers. The filtrate after the 1st spin of the RF supernatant through the $50 \mathrm{kDa}$ MWCO filter was used to isolate hydrolyzed monomers. This filtrate was brought to $\mathrm{pH} 7$ in a two-step process: it was first diluted in DMEM no serum media in $1: 1$ proportion $(500 \mu \mathrm{l}$ DMEM and $500 \mu \mathrm{l}$ of the filtrate) and spun for $22-24 \mathrm{~h}$ at $15000 \mathrm{~g}$. The 
resultant supernatant was collected and filtered through $30 \mathrm{kD}$ MWCO filter for $20 \mathrm{~min}$ at $15000 \mathrm{~g}$. The residue on the top of the filter was collected and the $\mathrm{pH}$ adjustment completed by adding $300 \mu \mathrm{l}$ DMEM to the top of the filter. To remove toxic oligomers induced by the $\mathrm{pH}$ transfer, this suspension was filtered again through a $50 \mathrm{kDa}$ MWCO filter at $15000 \mathrm{~g}$ for 20 min, and the filtrate was collected.

Isolation of ESO/CFs. Formation of ESO/CFs in $250 \mathrm{mM}$ $\mathrm{NaCl}, \mathrm{pH}$-adjusted water at $52{ }^{\circ} \mathrm{C}$ was monitored using dynamic light scattering (Malvern Zetasizer Nano) until the ESO/CF peak reached approx. $70 \%$ of total scattering intensity. Resulting ESO/CFs were separated from monomers using filtration through $50 \mathrm{kDa}$ MWCO filters.

\subsection{Cell viability assay}

The cytotoxicity of the various hewL aggregates was investigated using adenocarcinomic human alveolar basal epithelial cells (A549). Cell viability was assessed using a live/dead fluorescence assay. A549 cells were cultured in DMEM medium supplemented with $10 \%$ fetal bovine serum (Gibco), 1\% antibiotic-antimycotic $(100 \times)$ and $2 \mathrm{~g} \mathrm{l}^{-1} \mathrm{NaHCO}_{3}$. Cells were maintained in a $5 \% \mathrm{CO}_{2}$ humidified atmosphere at $37{ }^{\circ} \mathrm{C}$ and seeded at a density of $1.5 \times 10^{4}$ cells per well in 96 well plates. After 24 hours, the cell medium was replaced with DMEM without FBS and the plate incubated for another 24 hours. HewL aggregates were added to the cells and incubated for 4872 hours. Dilutions of all aggregates were chosen so that the same dilutions of the growth medium (50 or $250 \mathrm{mM} \mathrm{NaCl}$ at $\mathrm{pH} 2$ ) with the same concentrations of monomeric hewL did not affect cell viability. Cell medium was used as negative control and the apoptotic peptide toxin $\mathrm{KLAK}^{73}$ at 50-100 $\mu \mathrm{M}$ was used as positive toxicity control. After washing the cells twice in PBS, any dead cells were stained with $4 \mu \mathrm{M}$ of the membrane impermeable nuclear stain ethidium homodimer-1 (Life Technologies) for $1 \mathrm{~h}$ at $37^{\circ} \mathrm{C}$. Subsequently, live cells were stained with the cell permeant dye Hoechst 33342 (NucBlue, Life Technologies) for 30 minutes. Five non-overlapping regions were imaged from each well using an EVOS inverted fluorescence microscope at $10 \times$ magnification. NucBlue and ethidium homodimer fluorescence were imaged using a DAPI and RFP filter cube, respectively. Both fluorescence images were overlayed with brightfield images of the same region. Live/dead cell counting was performed using NIH ImageJ. Fluorescence and bright-field images in Fig. 4 were adjusted for contrast and intensity for display purposes.

\subsection{FPLC and SDS PAGE analysis}

For fast liquid protein chromatograph (FPLC) 1-2 mg of protein was dissolved in $500 \mu \mathrm{l}$ of DI water with $50 \mathrm{mM} \mathrm{NaCl}$, adjusted to $\mathrm{pH} 2$ with $\mathrm{HCl}$, as running buffer. Samples were injected into a Superdex 75 10/300 GL column (GE Healthcare) mounted on an ATKA PURE (GE Healthcare) FPLC. The column was typically rinsed with 2 column volumes of DI water, and then washed with running buffer for one column volume before injection of the sample. For separation, UV absorption was monitored at
$280 \mathrm{~nm}$ during column filtration and samples were fractionated into test tubes.

For reducing sodium dodecyl sulfate polyacrylamide gel electrophoresis (SDS PAGE), samples of fresh monomers, early-stage oligomers ESOs) and late-stage oligomers (LSOs) were prepared and isolated as described above. All samples were analyzed using 16.5\% gradient Tris-tricine gels (Criterion, Bio-Rad) with an SDS running buffer without glycine. An aliquot of each sample (2 $\mathrm{mg} \mathrm{ml}^{-1}$ ) was mixed with Tricine sample buffer (Bio-Rad) in the presence of $2 \%(\mathrm{v} / \mathrm{v}) 2$-mercaptoethanol (Fisher Scientific) in $1: 1$ ratio and boiled for 5 minutes at $90{ }^{\circ} \mathrm{C} .30 \mu \mathrm{l}$ of each sample was loaded onto the gel and run for about 1.5 hours at $105 \mathrm{~V}$. The gel was fixed in a solution of $40 \%$ methanol and $10 \%$ acetic acid for 30 minutes, stained for 2 hours using Coomassie blue (G-250 stain, Bio-Rad) for 1 hour, and was washed overnight with DI water.

\subsection{Antibody dot blots}

HewL monomer, and isolated RFs, ESO/CFs, LSOs and hydrolyzed monomer ( $2 \mu \mathrm{l}$ each) were spotted on nitrocellulose membranes (Thermofisher Pierce). HewL RFs absorbed onto the nitrocellulose membrane strongly while all other aggregates became diluted during washing steps. Using Ponceau staining, we adjusted the amount of protein deposited to obtain comparable total protein retained by the membrane. Membranes were blocked with $7 \%$ milk in $1 \times$ Tris-buffered saline (TBS) at room temperature for $1 \mathrm{~h}$. After three washing steps in $1 \times$ TBST (5 min each), membranes were incubated overnight at $4{ }^{\circ} \mathrm{C}$ with $1: 1000$ dilution of the amyloid oligomer antibody A11 (Novus Biologicals). Membranes were washed in $1 \times$ TBST for 3 times ( 5 min each) and were incubated in 1:1000 dilution of Ig goat anti-rabbit antibody (Southern biotech) for $1 \mathrm{~h}$ at room temperature. After $1 \mathrm{~h}$, membranes were washed in $1 \times$ TBST for 3 times and once in $1 \times$ TBS. Then, ECL western blotting substrate (Thermo Fisher Pierce) was added for detection. Sample photoluminescence was detected using a Chemidoc imager (Bio-Rad).

\subsection{Tinctorial profiles}

The fluorescence emission spectra of RFs, ESOs and LSOs relative to monomers were obtained using the fibril-selective dyes Thioflavin T, FSB and curcumin, ${ }^{59}$ as well as the oligomerselective dyes bis-ANS and Crystal Violet. ${ }^{62,63}$ Concentrations of dyes used were typically $15 \mu \mathrm{M}$ and aggregate concentrations were adjusted to $1 \mathrm{mg} \mathrm{ml}^{-1}$. Dye emissions for aggregates were excited at $445 \mathrm{~nm}$ for ThT, 377 (ESO) or $395 \mathrm{~nm}$ for FSB, $449 \mathrm{~nm}$ for curcumin, $395 \mathrm{~nm}$ for bis-ANS, and 583 (ESO) or $593 \mathrm{~nm}$ for $\mathrm{CV}$, respectively, and emission spectra collected using a spectrofluorometer (Fluoromax-4, Horiba Instruments, USA) using excitation/emission slit width of $5 \mathrm{~mm}$ each.

\section{Author contributions}

C. N., J. B., N. B., K. K., F. F. and G. S. all participated in the investigation and formal analysis of the data. They also reviewed and edited the manuscript. P. K. provided input to 
the methodology and resources. M. M. conceptualized the project, secured funding, provided resources and wrote the original draft.

\section{Conflicts of interest}

There are no conflicts to declare.

\section{Acknowledgements}

We would like to acknowledge support and helpful suggestions from Drs. Laura Blair and Shannon Hill with the immunostaining assays. We are also grateful for access to the Chemidoc Imager by Dr Kristina Schmidt's and the FPLC instrumentation of Dr Jianjun Pan. This research was funded, in part, by NIH grant 2R15GM097723-02.

\section{References}

1 F. Chiti and C. M. Dobson, Annu. Rev. Biochem., 2006, 75, 333-366.

2 T. P. J. Knowles, M. Vendruscolo and C. M. Dobson, Nat. Rev. Mol. Cell Biol., 2014, 15, 384-396.

3 D. Eisenberg and M. Jucker, Cell, 2012, 148, 1188-1203. 4 J. N. Buxbaum, Curr. Opin. Rheumatol., 2004, 16, 67-75.

5 C. M. Dobson, Trends Biochem. Sci., 1999, 24, 329-332.

6 M. Fändrich and C. M. Dobson, $E M B O$ J., 2002, 21, 5682-5690.

7 M. B. Pepys, Annu. Rev. Med., 2006, 57, 223-241.

8 E. N. Cline, M. A. Bicca, K. L. Viola and W. L. Klein, J. Alzheimer's Dis., 2018, 64, S567-S610.

9 U. Sengupta, A. N. Nilson and R. Kayed, EBioMedicine, 2016, 6, 42-49.

10 R. Kayed and C. A. Lasagna-Reeves, J. Alzheimer's Dis., 2013, 33, S67-S78.

11 E. K. Pickett, R. M. Koffie, S. Wegmann, C. M. Henstridge, A. G. Herrmann, M. Colom-Cadena, A. Lleo, K. R. Kay, M. Vaught, R. Soberman, D. M. Walsh, B. T. Hyman and T. L. Spires-Jones, J. Alzheimer's Dis., 2016, 53, 787-800.

12 F. Hefti, W. F. Goure, J. Jerecic, K. S. Iverson, P. A. Walicke and G. A. Krafft, Trends Pharmacol. Sci., 2013, 34, 261-266.

13 R. M. Koffie, M. Meyer-Luehmann, T. Hashimoto, K. W. Adams, M. L. Mielke, M. Garcia-Alloza, K. D. Micheva, S. J. Smith, M. L. Kim, V. M. Lee, B. T. Hyman and T. L. SpiresJones, Proc. Natl. Acad. Sci. U. S. A., 2009, 106, 4012-4017.

14 R. Tycko, Neuron, 2015, 86, 632-645.

15 W. Qiang, K. Kelley and R. Tycko, J. Am. Chem. Soc., 2013, 135, 6860-6871.

16 W. Close, M. Neumann, A. Schmidt, M. Hora, K. Annamalai, M. Schmidt, B. Reif, V. Schmidt, N. Grigorieff and M. Fändrich, Nat. Commun., 2018, 9, 699.

17 P. Liu, M. N. Reed, L. A. Kotilinek, M. K. O. Grant, C. L. Forster, W. Qiang, S. L. Shapiro, J. H. Reichl, A. C. A. Chiang, J. L. Jankowsky, C. M. Wilmot, J. P. Cleary, K. R. Zahs and K. H. Ashe, Cell Rep., 2015, 11, 1760-1771.
18 R. Kayed, I. Canto, L. Breydo, S. Rasool, T. Lukacsovich, J. Wu, R. Albay, A. Pensalfini, S. Yeung, E. Head, J. L. Marsh and C. Glabe, Mol. Neurodegener., 2010, 5, 57.

19 F. Hasecke, T. Miti, C. Perez, J. Barton, D. Schölzel, L. Gremer, C. S. R. Grüning, G. Matthews, G. Meisl, T. P. J. Knowles, D. Willbold, P. Neudecker, H. Heise, G. Ullah, W. Hoyer and M. Muschol, Chem. Sci., 2018, 9, 5937-5948.

20 S. Higashi, E. Iseki, R. Yamamoto, M. Minegishi, H. Hino, K. Fujisawa, T. Togo, O. Katsuse, H. Uchikado, Y. Furukawa, K. Kosaka and H. Arai, Brain Res., 2007, 1184, 284-294.

21 M. J. Guerrero-Muñoz, D. L. Castillo-Carranza, S. Krishnamurthy, A. A. Paulucci-Holthauzen, U. Sengupta, C. A. Lasagna-Reeves, Y. Ahmad, G. R. Jackson and R. Kayed, Neurobiol. Dis., 2014, 71, 14-23.

22 T. L. Spires-Jones, J. Attems and D. R. Thal, Acta Neuropathol., 2017, 134, 187-205.

23 F. Hasecke, C. Niyangoda, G. Borjas, J. Pan, G. Matthews, M. Muschol and W. Hoyer, Angew. Chem., Int. Ed., 2021, 60, 3016-3021.

24 D. Puzzo, R. Piacentini, M. Fá, W. Gulisano, D. D. Li Puma, A. Staniszewski, H. Zhang, M. R. Tropea, S. Cocco, A. Palmeri, P. Fraser, L. D'Adamio, C. Grassi and O. Arancio, eLife, 2017, 6, e26991.

25 S. I. A. Cohen, M. Vendruscolo, M. E. Welland, C. M. Dobson, E. M. Terentjev and T. P. J. Knowles, J. Chem. Phys., 2011, 135, 065105.

26 T. P. J. Knowles, C. A. Waudby, G. L. Devlin, S. I. A. Cohen, A. Aguzzi, M. Vendruscolo, E. M. Terentjev, M. E. Welland and C. M. Dobson, Science, 2009, 326, 1533-1537.

27 S. I. A. Cohen, M. Vendruscolo, C. M. Dobson and T. P. J. Knowles, in Amyloid Fibrils and Prefibrillar Aggregates, ed. D. E. Otzen, Wiley-VCH, Weinheim, Germany, 2013, pp. 183-209.

28 U. Sengupta, N. Puangmalai, N. Bhatt, S. Garcia, Y. Zhao and R. Kayed, Mol. Neurobiol., 2020, 57, 2741-2765.

29 S. Massimo, Curr. Protein Pept. Sci., 2010, 11, 343-354.

30 L. Breydo and V. N. Uversky, FEBS Lett., 2015, 589, 2640-2648.

31 G. M. Shankar, S. Li, T. H. Mehta, A. Garcia-Munoz, N. E. Shepardson, I. Smith, F. M. Brett, M. A. Farrell, M. J. Rowan, C. A. Lemere, C. M. Regan, D. M. Walsh, B. L. Sabatini and D. J. Selkoe, Nat. Med., 2008, 14, 837-842.

32 J. Lee, E. K. Culyba, E. T. Powers and J. W. Kelly, Nat. Chem. Biol., 2011, 7, 602-609.

33 T. R. Serio, A. G. Cashikar, A. S. Kowal, G. J. Sawicki, J. J. Moslehi, L. Serpell, M. F. Arnsdorf and S. L. Lindquist, Science, 2000, 289, 1317-1321.

34 D. L. Brody, H. Jiang, N. Wildburger and T. J. Esparza, Alzheimer's Res. Ther., 2017, 9, 62.

35 W. F. Goure, G. A. Krafft, J. Jerecic and F. Hefti, Alzheimer's Res. Ther., 2014, 6, 42.

36 T. Miti, M. Mulaj, J. D. Schmit and M. Muschol, Biomacromolecules, 2015, 16, 326-335.

37 J. Foley, S. E. Hill, T. Miti, M. Mulaj, M. Ciesla, R. Robeel, C. Persichilli, R. Raynes, S. Westerheide and M. Muschol, J. Chem. Phys., 2013, 139, 121901.

38 S. E. Hill, T. Miti, T. Richmond and M. Muschol, PLoS One, 2011, 6, e18171. 
39 S. E. Hill, J. Robinson, G. Matthews and M. Muschol, Biophys. J., 2009, 96, 3781-3790.

40 T. C. Dickson and J. C. Vickers, Neuroscience, 2001, 105, 99-107.

41 S. Han, M. Kollmer, D. Markx, S. Claus, P. Walther and M. Fändrich, Sci. Rep., 2017, 7, 43577.

42 J. C. Morris, M. Storandt, D. W. J. McKeel, E. H. Rubin, J. L. Price, E. A. Grant and L. Berg, Neurology, 1996, 46, 707-719.

43 C. A. Lemere, J. K. Blusztajn, H. Yamaguchi, T. Wisniewski, T. C. Saido and D. J. Selkoe, Neurobiol. Dis., 1996, 3, 16-32.

44 E. Head, I. T. Lott, D. M. Wilcock and C. A. Lemere, Curr. Alzheimer Res., 2016, 13, 18-29.

45 D. P. Perl, Mt. Sinai J. Med., 2010, 77, 32-42.

46 D. Hayne, S. Lim and P. Donnelly, Chem. Soc. Rev., 2014, 43, 6701-6715.

47 A. Abedini, A. Plesner, P. Cao, Z. Ridgway, J. Zhang, L.-H. Tu, C. T. Middleton, B. Chao, D. J. Sartori, F. Meng, H. Wang, A. G. Wong, M. T. Zanni, C. B. Verchere, D. P. Raleigh and A. M. Schmidt, eLife, 2016, 5, e12977.

48 R. Mishra, K. Sörgjerd, S. Nyström, A. Nordigården, Y.-C. Yu and P. Hammarström, J. Mol. Biol., 2007, 366, 1029-1044.

49 E. Frare, P. Polverino de Laureto, J. Zurdo, C. M. Dobson and A. Fontana, J. Mol. Biol., 2004, 340, 1153-1165.

50 G. Zandomeneghi, M. R. H. Krebs, M. G. McCammon and M. Fändrich, Protein Sci., 2004, 13, 3314-3321.

51 E. Cerf, R. Sarroukh, S. Tamamizu-Kato, L. Breydo, S. Derclaye, Y. F. Dufrêne, V. Narayanaswami, E. Goormaghtigh, J.-M. Ruysschaert and V. Raussens, Biochem. J., 2009, 421, 415-423.

52 J.-M. Ruysschaert and V. Raussens, Methods Mol. Biol., 2018, 1777, 69-81.

53 J. C. Stroud, C. Liu, P. K. Teng and D. Eisenberg, Proc. Natl. Acad. Sci. U. S. A., 2012, 109, 7717-7722.

54 L. Zhou and D. Kurouski, Anal. Chem., 2020, 92, 6806-6810.

55 A. Laganowsky, C. Liu, M. R. Sawaya, J. P. Whitelegge, J. Park, M. Zhao, A. Pensalfini, A. B. Soriaga, M. Landau, P. K. Teng, D. Cascio, C. Glabe and D. Eisenberg, Science, 2012, 335, 1228-1231.

56 M. I. Apostol, K. Perry and W. K. Surewicz, J. Am. Chem. Soc., 2013, 135, 10202-10205.

57 R. Kayed, E. Head, F. Sarsoza, T. Saing, C. Cotman, M. Necula, L. Margol, J. Wu, L. Breydo, J. Thompson, S. Rasool, T. Gurlo, P. Butler and C. Glabe, Mol. Neurodegener., 2007, 2, 18.

58 R. Kayed, E. Head, J. L. Thompson, T. M. McIntire, S. C. Milton, C. W. Cotman and C. G. Glabe, Science, 2003, 300, 486-489.
59 C. Condello, T. Lemmin, J. Stöhr, M. Nick, Y. Wu, A. M. Maxwell, J. C. Watts, C. D. Caro, A. Oehler, C. D. Keene, T. D. Bird, S. G. van Duinen, L. Lannfelt, M. Ingelsson, C. Graff, K. Giles, W. F. DeGrado and S. B. Prusiner, Proc. Natl. Acad. Sci. U. S. A., 2018, 115, E782-E791.

60 B. Bolognesi, J. R. Kumita, T. P. Barros, E. K. Esbjorner, L. M. Luheshi, D. C. Crowther, M. R. Wilson, C. M. Dobson, G. Favrin and J. J. Yerbury, ACS Chem. Biol., 2010, 5, 735-740.

61 M. Lindgren and P. Hammarström, FEBS J., 2010, 277, 1380-1388.

62 N. D. Younan and J. H. Viles, Biochemistry, 2015, 54, 4297-4306.

63 J. Barton, D. S. Arias, C. Niyangoda, G. Borjas, N. Le, S. Mohamed and M. Muschol, Biomolecules, 2019, 9, 539.

64 N. P. Harte, I. Klyubin, E. K. McCarthy, S. Min, S. A. Garrahy, Y. Xie, G. P. Davey, J. J. Boland, M. J. Rowan and K. H. Mok, J. Biol. Chem., 2015, 290, 28343-28352.

65 M. N. N. Vieira, L. Forny-Germano, L. M. Saraiva, A. Sebollela, A. M. B. Martinez, J.-C. Houzel, F. G. De Felice and S. T. Ferreira, J. Neurochem., 2007, 103, 736-748.

66 G. Fusco, S. W. Chen, P. T. F. Williamson, R. Cascella, M. Perni, J. A. Jarvis, C. Cecchi, M. Vendruscolo, F. Chiti, N. Cremades, L. Ying, C. M. Dobson and A. De Simone, Science, 2017, 358, 1440-1443.

67 S. A. Kotler, J. R. Brender, S. Vivekanandan, Y. Suzuki, K. Yamamoto, M. Monette, J. Krishnamoorthy, P. Walsh, M. Cauble, M. M. B. Holl, E. N. G. Marsh and A. Ramamoorthy, Sci. Rep., 2015, 5, 11811.

68 B. Mannini, E. Mulvihill, C. Sgromo, R. Cascella, R. Khodarahmi, M. Ramazzotti, C. M. Dobson, C. Cecchi and F. Chiti, ACS Chem. Biol., 2014, 9, 2309-2317.

69 A. R. A. Ladiwala, J. Litt, R. S. Kane, D. S. Aucoin, S. O. Smith, S. Ranjan, J. Davis, W. E. Van Nostrand and P. M. Tessier, J. Biol. Chem., 2012, 287, 24765-24773.

70 D. R. Booth, M. Sunde, V. Bellotti, C. V. Robinson, W. L. Hutchinson, P. E. Fraser, P. N. Hawkins, C. M. Dobson, S. E. Radford, C. F. F. Blake and M. B. Pepys, Nature, 1997, 385, 787-793.

71 K. W. Tipping, T. K. Karamanos, T. Jakhria, M. G. Iadanza, S. C. Goodchild, R. Tuma, N. A. Ranson, E. W. Hewitt and S. E. Radford, Proc. Natl. Acad. Sci. U. S. A., 2015, 112, 5691-5696.

72 L. R. Wilken and Z. L. Nikolov, Biotechnol. Prog., 2006, 22, 745-752.

73 H. M. Ellerby, W. Arap, L. M. Ellerby, R. Kain, R. Andrusiak, G. D. Rio, S. Krajewski, C. R. Lombardo, R. Rao, E. Ruoslahti, D. E. Bredesen and R. Pasqualini, Nat. Med., 1999, 5, 1032-1038. 